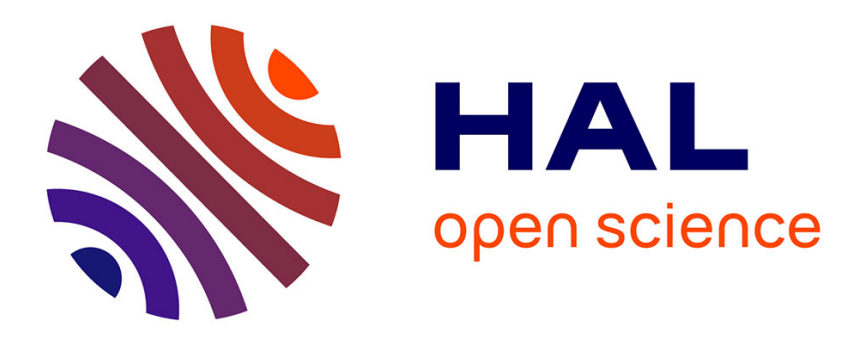

\title{
Sur la constitution de la charge électrique à la surface d'un électrolyte
}

\author{
M. Gouy
}

\section{To cite this version:}

M. Gouy. Sur la constitution de la charge électrique à la surface d'un électrolyte. J. Phys. Theor. Appl., 1910, 9 (1), pp.457-468. 10.1051/jphystap:019100090045700 . jpa-00241565

\section{HAL Id: jpa-00241565 https://hal.science/jpa-00241565}

Submitted on 1 Jan 1910

HAL is a multi-disciplinary open access archive for the deposit and dissemination of scientific research documents, whether they are published or not. The documents may come from teaching and research institutions in France or abroad, or from public or private research centers.
L'archive ouverte pluridisciplinaire HAL, est destinée au dépôt et à la diffusion de documents scientifiques de niveau recherche, publiés ou non, émanant des établissements d'enseignement et de recherche français ou étrangers, des laboratoires publics ou privés. 


\section{SUR LA CONSTITUTION \\ DE LA GHARGE ÉLECTRIQUE A LA SURFAGE D'UN ÉLECTROLYTE(1);}

Par M. GOUY.

1. On admet généralement que, lorsqu'on électrise un électrolyte, il s'accumule à sa surface des ions qui rendent le potentiel uniforme dans toute la masse liquide. Cette idée de la charge purement superficielle, qui paraît d'abord très plausible, conduit pourtant à des contradictions singulières, dont voici un exemple.

Considérons deux condensateurs identiques, dont les armatures extérieures sont formées par une solution aqueuse où ils sont plongés. Soient $Q$ et $-Q$ les charges des armatures intérieures, $V_{1}$ et $V_{2}$ leurs potentiels, $p$ la pression osmotique. Par une opération isotherme et réversible faisons varier $\mathrm{Q}$ en fournissant le travail $\left(\mathrm{V}_{1}-\mathrm{V}_{2}\right) d \mathrm{Q}$, et faisons varier la concentration en introduisant le volume $d v \mathrm{du}$ dissolvant, et fournissant le travail - pdv. La variation de l'énergie utilisable sera :

$$
\left(\mathrm{V}_{1}-\mathrm{V}_{2}\right) d Q-p d v .
$$

Comme c'est une différentielle exacte, il vient :

$$
\frac{\partial}{\partial v}\left(\mathrm{~V}_{1}-\mathrm{V}_{2}\right)=-\frac{\partial p}{\partial Q} \text {. }
$$

Mais si $Q=0$, on a $V_{1}=V_{2}$ par symétrie, et par suite :

$$
\frac{\partial p}{\partial Q}=0 \text {. }
$$

Ainsi une petite charge ne fait pas varier la concentration de l'électrolyte dans son intérieur, aux quantités du $2^{\mathrm{e}}$ ordre près. Cependant, s'il y avait charge superficielle, il y aurait accumulation, surla surface de l'électrolyte, de cathions d'un côté, et d'anions de l'autre, soustraits à la masse liquide, qui serait ainsi appauvrie.

2. Un examen plus attentif montre que la charge ne peut pas être purement superficielle. En effet, les ions sont soumis, en outre des actions électriques qui tendent à les accumuler à la surface, à la dif-

(1) Communication faite à la Société française de physique : séance du 18 févri er 1910.

J. de Phys., $4^{\mathrm{e}}$ série, t. IX. (Juin 1910.) 
fusion qui tend à rétablir l'homogénéité qui existait avant la charge (1). Il en résulte un état d'équilibre, que nous allons calculer en unités électrostatiques.

Soient $\nu_{c}, v_{c}^{\prime} \ldots$, et $\nu_{a}, v_{a}^{\prime} \ldots$, les valences des cathions et des anions; $\mathrm{N}_{c}, \mathrm{~N}_{\prime}^{\prime}, \ldots$ et $\mathrm{N}_{a}, \mathrm{~N}_{a}, \ldots$ sont les nombres d'ions-grammes par unité de volume, loin de la surface. A la distance $x$ de la surface, ces nombres deviennent $\mathrm{N}_{c} \mathrm{U}_{c}, \mathrm{~N}^{\prime} \mathrm{U}_{c}^{\prime}, \ldots$ et $\mathrm{N}_{a} \mathrm{U}_{a}, \mathrm{~N}^{\prime}{ }_{a} \mathrm{U}^{\prime}{ }_{a}, \ldots$ Soient $p$ la densité électrique au point $(x)$ et $m$ la charge de 1 gramme d'ionshydrogène. On a :

(2) $\rho=m\left(\mathbf{N}_{c} \mathrm{U}_{c^{\nu} c}+\mathbf{N}_{c}{ }_{c} \mathrm{U}^{\prime{ }^{\nu^{\prime}}}{ }_{c}+\ldots-\mathrm{N}_{a} \mathrm{U}_{a^{\nu} a}-\mathrm{N}_{a}^{\prime} \mathrm{U}^{\prime}{ }_{a{ }^{\nu^{\prime}}}{ }_{a}-\ldots\right)$

Les forces étant comptées positivement dans le sens des $x$ croissants, considérons l'équilibre des cathions de la première espèce contenus dans la couche $d x$. Soit $\mathrm{K}$ le pouvoir inducteur du liquide, qui est une constante, la solution étant étendue. Une masse +1 placée au point $(x)$ serait sollicitée par la force :

$$
\frac{2 \pi}{\mathrm{K}}\left(\int_{0}^{x} \rho d x-\int_{x}^{\infty} \rho d x\right)+\mathrm{F},
$$

F étant la force produite par les charges extérieures, qui sera constante dans l'intervalle très petit que nous considérons $\left({ }^{2}\right)$. Comme, à distance de la surface, il n'y a plus de champ, nous avons :

$$
\frac{2 \pi}{\mathrm{K}} \int_{0}^{\infty} \mathrm{p} d x+\mathrm{F}=0,
$$

et par suite en posant :

$$
q=\int_{x}^{\infty} \rho d x
$$

nous avons, par unité de surface, pour la force électrique exercée sur nos cathions,

$$
-\frac{4 \pi q}{\mathrm{~K}} m \mathrm{~N}_{c} \mathrm{U}_{c^{{ }}{ }_{c}} d x
$$

(1) Si, avant la charge, les ions sont distribués inégalement, en raison, par exemple de forces non électriques qui les attirent vers la surface, cette distribution modifiera celle que nous allons calculer; mais nous excluons ce cas pour le moment.

(2) La surface est regardée comme plane, ou du moins son rayon de courbure est très grand vis-à-vis de l'épaisseur de la couche où $p$ est sensible. 
CHARGE ÉLECTRIQUE A LA SURFACE D'UN ÉLECTROLYTE $4 \mathbf{4 9}$

Pour calculer l'effet de la diffusion, nous pouvons imaginer que la couche $d x$ est enfermée entre deux parois semi-perméables qui arrêtent seulement les cathions considérés. La résultante des deux pressions osmotiques exercées sur cette couche est $-\mathrm{RTN}_{c} \frac{d \mathrm{U}_{c}}{d x} d x$, et l'équation d'équilibre s'écrit ( $\left.{ }^{1}\right)$ :

$$
\frac{4 \pi q m}{\mathrm{KRT}}=-\frac{1}{\mathrm{U}_{c^{v} c}} \frac{d \mathrm{U}_{c}}{d x}=-\frac{d}{d x} \log \frac{1}{\mathrm{U}_{c}^{c_{c}}}
$$

on a de même :

(4 bis)

$$
\frac{4 \pi q m}{\mathrm{KRT}}=\frac{1}{\mathrm{U}_{a^{1} a}} \frac{d \mathrm{U}_{a}}{d x}=\frac{d}{d x} \log \mathrm{U}_{a^{v_{a}}}
$$

Comme à l'infini, tous les U sont égaux à 1, on a, en écrivant toutes les équations telles que (4) et (4 bis),

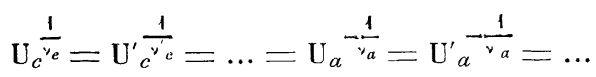

et en ajoutant membre à membretoutes les équations $(4)$ et $\left(\begin{array}{ll}4 & b i s\end{array}\right)$,

$$
\begin{aligned}
& \frac{d}{d x}\left(\mathrm{~N}_{c} \mathrm{U}_{c}+\mathrm{N}^{\prime} \mathrm{U}^{\prime}{ }_{c}+\ldots+\mathrm{N}_{a} \mathrm{U}_{a}+\mathrm{N}^{\prime}{ }_{a} \mathrm{U}^{\prime}{ }_{a}+\ldots\right) \\
& =-\frac{4 \pi q m}{\mathrm{KRT}}\left(\mathrm{N}_{c} \mathrm{U}_{e^{\nu} c}+\mathrm{N}_{c}{ }_{c} \mathrm{U}^{\prime} c^{\nu^{\prime}}{ }_{c}+\ldots-\mathrm{N}_{a} \mathrm{U}_{a^{\nu} a}-\mathrm{N}_{a}{ }_{a} \mathrm{U}^{\prime} a^{\nu^{\prime}} a-\ldots\right) \text {. }
\end{aligned}
$$

Mais, d'après (2), la parenthèse du deuxième membre

$$
=\frac{\rho}{m}=-\frac{1}{m} \frac{d q}{d x} .
$$

Il vient donc :

$$
\mathrm{N}_{c} \mathrm{U}_{c}+\mathrm{N}_{c}^{\prime} \mathrm{U}_{c}^{\prime}+\ldots+\mathrm{N}_{a} \mathrm{U}_{a}+\mathrm{N}^{\prime}{ }_{a} \mathrm{U}^{\prime}{ }_{a}+\ldots=\frac{2 \pi}{\mathrm{KRT}} q^{2}+\mathrm{C}^{\mathrm{t} \bullet} .
$$

Comme pour $x$ très grand, on a $q=0$, et tous les $\mathrm{U}$ égaux à 1 , il vient :

(6) $\mathrm{N}_{c}\left(\mathrm{U}_{c}-1\right)+\mathrm{N}_{c}{ }_{c}\left(\mathrm{U}_{c}^{\prime}-1\right)+\ldots+\mathrm{N}_{a}\left(\mathrm{U}_{a}-1\right)+\mathrm{N}^{\prime}{ }_{a}\left(\mathrm{U}^{\prime}{ }_{a}-1\right)+\ldots=\frac{2 \pi}{\mathrm{KRT}} q^{2}$

3. Les équations (5) et (6) donneront tous les $\mathrm{U}$ en fonction de $q$. On peut immédiatement s'en servir pour calculer les valeurs $U_{\text {cos }}$

(1) Les logarithmes sont népériens, 
$\mathrm{U}_{a_{0}}$, à la surface en fonction de la charge $q_{0}$ par unité de surface, l'indice 0 désignant partout les valeurs prises à la surface.

Les équations (5) nous montrent immédiatement ce qui suit :

$1^{\circ}$ Si les cathions ont même valence, leurs concentrations restent partout proportionnelles et de même pour les anions. Si les ions ont des valences inégales, ils sont d'autant plus condensés ou raréfiés dans les couches voisines de la surface que leurs valences sont plus grandes ;

$2^{\circ}$ Les concentrations des ions varient de la manière qui correspond à l'équilibre chimique avec des molécules non dissociées de concentration constante. Ainsi, pour un cathion et un anion de même valence, le produit des concentrations $\mathrm{N}_{c} \mathrm{U}_{c} \times \mathrm{N}_{a} \mathrm{U}_{a}$ est constant, d'après $(\breve{3})$.

Il devait en être ainsi, car le principe de Carnot exige qu'on ait équilibre chimique aussi bien que physique en chaque point. Sans cela, en effet, dans certaines couches, les ions formeraient des molécules, ce qui entraînerait une circulation perpétuelle d'ions et de molécules.

11 ressort de ce qui précède que, pour les solutions aqueuses, où les ions propres de l'eau sont monovalents et extrêmement rares, on pourra en faire abstraction, puisqu'ils seront partout en proportion négligeable. C'est ce que nousferons désormais, et nous considérerons le cas d'un seul cathion et d'un seul anion, tous les $\mathrm{N}$ étant nuls, sauf $\mathrm{N}_{c}$ et $\mathrm{N}_{a}$.

4. Posons

$$
\mathrm{C}=\mathrm{N}_{c^{v} c}=\mathrm{N}_{a^{v} a} \text {. }
$$

L'équation (6) s'écrit

$$
\frac{\mathrm{U}_{c}-1}{v_{c}}+\frac{\mathrm{U}_{a}-1}{v_{a}}=\frac{2 \pi}{\operatorname{CKRT}} q^{2} .
$$

Posons

$$
\frac{v_{c}}{v_{a}}=n, \quad \text { et } \quad \frac{1}{z}=\mathrm{U}_{c} .
$$

On aura, d'après (ら),

$$
\mathrm{U}_{a}=z^{n} .
$$

L'équation (6 bis) s'écrit:

$$
z^{n+1}-z\left(n+1+\frac{2 \pi v_{a}}{\mathrm{CKRT}} q^{2}\right)+n=0 .
$$


CHARGE ÉLEGTRIQUE A LA SURFACE D'UN ÉLECTROLYTE 461

On voit aisément que l'équation en $z$ n'admet que deux racines convenant à la question, c'est-à-dire réelles, positives et se réduisant à l'unité pour $q=0$; ces racines sont l'une $>1$ et l'autre $<1$. On choisira celle qui convient pour que $\mathrm{U}_{c}-1$ soit du signe de $q_{0}$, Ainsi $z$ est déterminé sans ambiguïté.

Calculons $x$ en fonction de $z$. On a :

$$
x=\int_{g_{\circ}}^{q} \frac{d x}{d q} d q=\int_{q}^{q_{0}} \frac{d q}{\rho}=\frac{1}{m \mathrm{C}} \int_{q}^{q_{0}} \frac{d q}{\mathrm{U}_{c}-\mathrm{U}_{a}}=\frac{1}{m \mathrm{C}} \int_{q}^{q_{0}} \frac{z}{1-z^{n+1}} d q .
$$

Mais, d'après (7), on a :

d'où :

$$
d q=\frac{n}{q} \frac{\mathrm{GKRT}}{4 \pi v_{a}} \frac{z^{n+1}-1}{z^{2}} d z
$$

$$
x=\frac{\mathrm{KRT}}{4 \pi m v_{c}} \int_{z_{0}}^{z} \frac{d z}{q z}
$$

$q$ étant exprimé en fonction de z après (7), on aura ainsi l'expression de $x$ en fonction de $z$.

5. Examinons quelques conséquences de ce qui précède.

Supposons que la charge $q_{0}$ vienne à varier, en sorte que $z_{0}$ devienne $\boldsymbol{z}_{0}^{\prime}$, et que $x$ devienne $x^{\prime}$; on aura:

$$
x^{\prime}=x-\frac{\mathrm{KRT}}{4 \pi m v_{c}} \int_{z_{0}}^{z_{0}^{\prime}} \frac{d z}{q z}=x+\mathrm{C}^{\mathrm{te}} .
$$

Ainsi, lorsqu'on aura tracé les courbes représentatives de $\mathrm{U}_{c}$ et de $\mathrm{U}_{a}$ en fonction de $x$, pour une certaine valeur $q_{0}$, il suffira, si cette valeur vient à changer, de donner aux courbes une translation parallèle à l'axe des $x$ jusqu'à ce que, à la surface, on ait les valeurs de $\mathrm{U}_{c}$ et de $\mathrm{U}_{a}$ qui conviennent, d'après (5) et (6), à la nouvelle valeur de la charge.

Ainsi, avec une certaine charge, on aura, à une certaine profondeur, le même état qu'on aurait à une profondeur moindre, avec une charge plus faible.

Examinons maintenant comment varie $x$, si l'on donne d'autres valeurs à la concentration C. Supposons que C soit multiplié par $\beta^{2}$, 
sans que $z_{0}$ varie, ce qui implique que $q_{0}$ sera multiplié par $\beta$, ainsi que là valeur de $q$ qui correspond à un $z$ quelconque. Par suite $x$ sera multiplié $\operatorname{par} \frac{1}{\beta}$.

Ainsi, pour passer des courbes de $\mathrm{U}_{c}$ et de $\mathrm{U}_{a}$ relatives à une certaine concentration à celles relatives à une autre concentration, il faut réduire les abscisses dans le rapport inverse des racines carrées des concentrations, et faire ensuite glisser les courbes comme plus haut, jusqu'à ce que $q_{0}$ ait sa valeur exacte. La charge est donc d'autant plus localisée au voisinage de la surface que la concentration (en ions) est plus grande.

6. L'expression (8) se calcule aisément quand le rapport $n$ des valences est 1, 2 ou $\frac{1}{2}$. Pour $n=1$, on trouve :

$$
x=\frac{1}{2 \sqrt{2} m} \sqrt{\frac{\mathrm{KRT}}{\mathrm{G} \pi v_{c}}}\left\{\log \frac{\sqrt{\mathrm{U}_{c 0}}-1}{\sqrt{\mathrm{U}_{c}}-1}-\log \frac{\sqrt{\mathrm{U}_{c 0}}+1}{\sqrt{\mathrm{U}_{c}}+1}\right\},
$$

d'où il vient, en désignant par $\alpha$ le coefficient de la parenthèse,

$$
\mathrm{U}_{c}=\left\{\left.\frac{\frac{\sqrt{\mathrm{U}_{c 0}}+1}{\sqrt{\mathrm{U}_{c 0}}-1} e^{\frac{x}{\alpha}}+1}{\frac{\sqrt{\mathrm{U}_{c 0}}+1}{\sqrt{\mathrm{U}_{c 0}}-1} e^{\frac{x}{\alpha}}-1}\right|^{2}=\frac{1}{\mathrm{U}_{a}} .\right.
$$

Si l'on a $n=2$, on trouve:

(11) $x=\frac{1}{2 \sqrt{3} m} \sqrt{\frac{\mathrm{KRT}}{\mathrm{C} \pi v_{c}}}\left\{\log \frac{\sqrt{2 \mathrm{U}_{c 0}+1}-\sqrt{3}}{\sqrt{2 \mathrm{U}_{c}+1}-\sqrt{3}}-\log \frac{\sqrt{2 \mathrm{U}_{c 0}+1}+\sqrt{3}}{\sqrt{2 \mathrm{U}_{c}+1}+\sqrt{3}}\right\}$,

et par suite, en désignant par $\alpha^{\prime}$ le coefficient de la parenthèse,

$$
\mathrm{U}_{c}=\frac{3}{2}\left\{\frac{\frac{\sqrt{2 \mathrm{U}_{c 0}+1}+\sqrt{3}}{\sqrt{2 \mathrm{U}_{c 0}+1}-\sqrt{3}} e^{\frac{x}{\alpha^{\prime}}+1}}{\frac{\sqrt{2 \mathrm{U}_{c 0}+1}+\sqrt{3}}{\sqrt{2 \mathrm{U}_{c 0}+1}-\sqrt{3}} e^{\frac{x}{r^{\prime}}}-1}\right\}^{2}-\frac{1}{2}=\frac{1}{\mathrm{U}_{a}^{2}} .
$$

Pour $n=\frac{1}{2}$, on permutera dans (11) et (12) les indices $c$ et $a$.

7. Centre de la gravite de la charge. -- Soit $\varepsilon$ sa distance à la sur- 
CHARGE ÉLECTRIQUE A LA SURFAGE D'UN ÉLEGTROLYTE 463 face. On a par définition :

$$
\varepsilon=\frac{1}{q_{0}} \int_{0}^{\infty} x_{\uparrow} d x=-\frac{1}{q_{0}} \int_{0}^{\infty} x \frac{d q}{d x} d x
$$

En intégrant par parties, et remarquant que $q x$ tend vers zéro quand $x$ croît sans limite $\left({ }^{1}\right)$, on a, d'après $(8)$,

$$
\text { (12) } \varepsilon=\frac{1}{q_{0}} \int_{0}^{\infty} q d x=\frac{1}{q_{0}} \frac{\mathrm{KRT}}{4 \pi m v_{c}} \int_{z_{0}}^{1} \frac{d z}{z}=\frac{1}{q_{0}} \frac{\mathrm{KRT}}{4 \pi m v_{c}} \log \mathrm{U}_{c_{0}}=-\frac{1}{q_{0}} \frac{\mathrm{KRT}}{4 \pi m v_{a}} \log \mathrm{U}_{a_{0}} .
$$

La connaissance de $\varepsilon$ est très utile, car la charge agit, au point de vue du potentiel, comme si elle était concentrée en une couche infiniment mince, en son centre de gravité.

Nous avons vu, en effet, au numéro 2, que la force qui agirait sur une masse +1 aurait pour valeur, en chaque point, $-\frac{4 \pi q}{\mathrm{~K}}$. Soit $\left(x_{1}\right)$ un point.éloigné de la surface, où le potentiel est $\mathrm{V}_{1}$, et soit $\mathrm{V}_{0}$ le potentiel à la surface. On a donc :

$$
\mathrm{V}_{1}-\mathrm{V}_{0}=\frac{4 \pi}{\mathrm{K}} \int_{0}^{x_{1}} q d x
$$

ou, en remplaçant $d x$ par sa valeur tirée de $(8)$,

$$
\mathrm{V}_{1}-\mathrm{V}_{0}=\frac{\mathrm{RT}}{m v_{c}} \int_{z_{0}}^{1} \frac{d z}{z}=\frac{\mathrm{RT}}{m v_{c}} \log \mathrm{U}_{c 0}=\frac{4 \pi}{\mathrm{K}} \varepsilon q_{0}
$$

ce qui est conforme à l'énoncé.

8. Appauvrissement de l'électrolyte. - Considérons un plan parallèle à la surface, à la distance $x_{1}$, assez grande pour que l'électrolyte n'y diffère plus sensiblement de ce qu'il est à l'intérieur. Si l'électrolyte restait homogène, le nombre des cathions-grammes compris entre la surface et ce plan serait $\mathrm{N}_{c} x_{1}$ par unité de surface. En réalité ce nombre est $\int_{0}^{x_{1}} \mathrm{~N}_{c} \mathrm{U}_{c} d x$. En posant:

$$
n_{c}=\int_{0}^{x_{1}} \mathrm{~N}_{c} \mathrm{U}_{c} d x-\mathrm{N}_{c} x_{1}=\mathrm{N}_{c} \int_{0}^{x_{1}}\left(\mathrm{U}_{c}-1\right) d x
$$

(1) Nous verrons en effet que $q$ finit par être proportionnel à $e^{-\frac{x}{a}}(a$ constante). 
$n_{c}$ représentera donc le nombre des cathions-grammes qui, du fait de la charge, se trouvent attirés vers chaque unité de surface et appauvrissent ainsi la masse de l'électrolyte ( $\left.{ }^{1}\right)$.

On aura la même formule pour les anions, en remplacant l'indice $c \operatorname{par} a$.

Remarquons d'abord que l'on_a,

$$
q_{0}=m\left(\mathrm{~N}_{c^{v} c} \int_{0}^{x_{1}} \mathrm{U}_{c} d x-\mathbf{N}_{a^{v} a} \int_{0}^{x_{1}} \mathrm{U}_{a} d x\right)=m\left(n_{c^{\nu} c}-n_{a^{v} a}\right) .
$$

Nous avons aussi, d'après (6 bis),

$$
n_{c}+n_{a}=\mathrm{C} \int_{0}^{x_{1}}\left(\frac{\mathrm{U}_{c}-1}{v_{c}}+\frac{\mathrm{U}_{a}-1}{v_{a}}\right) d x=\frac{2 \pi}{\mathrm{KRT}^{\prime}} \int_{0}^{x_{1}} q^{2} d x,
$$

et d'après (8), en remarquant que $z=1$ au point $\left(x_{1}\right)$, il vient :

$$
n_{c}+n_{a}=\frac{1}{2 m v_{c}} \int_{z_{0}}^{1} q \frac{d z}{z} .
$$

Si les valences sont égales, l'équation (14) donne:

$$
n_{c}+n_{a}=\frac{1}{m} \sqrt{\frac{\mathrm{CKRT}}{2 \pi v^{3} c}}\left(\sqrt{\mathrm{U}_{c 0}}+\sqrt{\mathrm{U}_{a 0}}-2\right) .
$$

Si l'on a $n=2$, il vient :

$$
\begin{aligned}
n_{c}+n_{a}=\frac{1}{4 m} \sqrt{\frac{\mathrm{CKRT}}{\pi y^{3}}}\left\{2 \sqrt{2 \mathrm{U}_{c 0}+1}-2 \sqrt{3}+\frac{1}{\sqrt{2 \mathrm{U}_{c 0}+1}-1}\right. \\
\left.\quad-\frac{1}{\sqrt{3}-1}+\frac{1}{\sqrt{2 \mathrm{U}_{c 0}+1}+1}-\frac{1}{\sqrt{3}+1}\right\} .
\end{aligned}
$$

Pour $n=\frac{1}{2}$, on remplacera l'indice $c$ par $a$.

9. Charge modérée ou pètite. - Les formules se simplifient beaucoup, quelles que soient les valences, si $\mathrm{U}_{c 0}$ et $\mathrm{U}_{a_{0}}$ sont très voisins de l'unité. Pour des solutions aqueuses centinormales (en ions) ou

(1) Il faut remarquer que, de ce fait, la quantité du dissolvant qui est comprise entre la surface et le plan $\left(x_{l}\right)$ se trouve un peu modifiée. Les données manquent pour tenir compte de cette petite complication. 
GHARGE ÉLEGTRIQUE A LA SURFACE D'UN ÉLEGTROLYTE 463 plus concentrées, cela aura lieu dès que $q_{0}$ sera inférieur à 10 unités électrostatiques, ce qui est une charge modérée de condensateur.

Posons :

$$
\delta=\mathrm{U}_{c}-1 .
$$

Nous avons :

$$
\mathrm{U}_{a}=\mathrm{U}_{c}^{-n}=(1+\delta)^{-n}=1-n \delta+\frac{n(n+1)}{1.2} \delta^{2} \ldots
$$

L'équation (6 $6 i s)$ s'écrit en traitant ò comme un infiniment petit;

d'où :

$$
\delta=q \sqrt{\frac{4 \pi v_{c}}{\operatorname{CKRT}(n+1)}}
$$

$$
\frac{\mathrm{U}_{c}-1}{v_{c}}=-\frac{\mathrm{U}_{a}-1}{v_{a}}=q \sqrt{\frac{4 \pi}{\operatorname{CKRT}\left(v_{c}+v_{a}\right)}} .
$$

On a :

$$
\frac{d z}{z}=\left(-1+\delta-\delta^{2}+\ldots\right) d \delta,
$$

et par suite, d'après $(8)$,

$$
x=\frac{1}{2 m} \sqrt{\frac{\mathrm{KRT}}{\mathrm{C} \pi\left(\nu_{c}+\nu_{a}\right)}} \log \frac{q_{0}}{q} .
$$

En posant :

$$
a=\frac{1}{2 m} \sqrt{\frac{\mathrm{KRT}}{\mathrm{C} \pi\left(\nu_{c}+v_{a}\right)}}
$$

il vient donc :

$$
q=q_{0} e^{-\frac{x}{a}} \quad \text { et } \quad \rho=\frac{q_{0}}{a} e^{-\frac{x}{a}}
$$

D'après (1 tion plus loin, en tenant compte des termes en $\delta^{2}$, il vient:

(18) $n_{c}+n_{a}=\frac{\delta_{0}^{2}}{4 m} \sqrt{\frac{\operatorname{CKRT}(n+1)}{4 \pi c_{c}^{3}}}=\frac{q_{0}^{2}}{2 m} \sqrt{\frac{\pi}{\operatorname{CKRT}\left(\nu_{c}+v_{a}\right)}}$.

De la sorte se trouve expliqué le résultat paradoxal trouvé au début (1).

Ainsi une charge moyenne ou petite consiste en une légère condensation des ions d'un certain signe, et une légère raréfaction des ions de signe opposé, toutes deux s'annulant asymptotiquement quand

(1) On peut vérifier aussi que, en tenant compte de (18), l'équation (1) est toujours satisfaite pour des solutions étendues. 
on s'éloigne de la surface. La compensation se fait quant au nombre des ions, et la masse intérieure n'est pas appauvrie.

Il résulte de ce que nous avons vu que cela sera encore vrai pour une forte charge, mais seulement aux profondeurs où $q$ devient assez petit.

Lá distance $\varepsilon$ du centre de gravité à la surface est égale à $a$, ce qui donne en microns, pour des solutions aqueuses d'ions monovalents (1).

Solution décinormale (en ions)........... $0^{\mu}, 00096$

Solution millinormale (en ions) .......... 0,0096

Eau pure de Kohlrausch............. 1,01

Les intervalles que nous avons à considérer sont donc bien supérieurs aux intervalles moléculaires, dès que la solution est étendue, car on sait qu'une molécule d'eau occupe le volume d'un cube d'environ $0^{\mu}, 0003$ de côté.

10. Charge très grande. - Supposons qu'une des quantités U, par exemple $\mathrm{U}_{c}$, ait une grande valeur. On a sensiblement d'après (6 bis),

$$
\mathrm{U}_{c}=\frac{2 \pi v_{c}}{\operatorname{CKRT}} q^{2}
$$

et

$$
\rho=m \mathrm{CU}_{c}=\frac{2 \pi m \nu_{c}}{\mathrm{KRT}} q^{2},
$$

d'où :

et

$$
x=\int_{q}^{q_{0}} \frac{d q}{\rho}=\frac{\mathrm{KRT}}{2 \pi m v_{c}} \int_{q}^{q_{0}} \frac{d q}{q^{2}}=\frac{\mathrm{KRT}}{2 \pi m v_{c}}\left(\frac{1}{q}-\frac{1}{q_{0}}\right),
$$

$$
\mathrm{U}_{c}=\frac{\frac{2 \pi v_{c}}{\mathrm{CKRT}}}{\left(\frac{2 \pi m y_{c}}{\mathrm{KRT}} x+\frac{1}{q_{0}}\right)^{2}}(2)
$$

On voit que, pour une valeur donnée de $\frac{q_{0}}{q}, x$ tend vers zéro, quand

(1) Données : $\mathrm{K}=80 ; \mathrm{R}=8,32 \cdot 10^{7} ; \mathrm{T}=291 ; m=2,90 \cdot 10^{14} ; \mathrm{C}$ vaut $10^{-4}$; pour la solution déci-normale et $0,9 \cdot 10^{-10}$ pour l'eau pure.

(2) Si $\mathrm{U}_{a}$ était très grand au lieu de $\mathrm{U}_{c}$, il faudrait remplacer l'indice $c$ par $a$, et changer le signe du deuxième membre dans l'expression de $x$. 
CHARGE ÉLEGTRIQUE A LA SURFAGE D'UN ÉLEGTROLYTE 467 la charge croît sans limite. L'équation (12) montre que, en même temps, $\varepsilon$ tend vers zéro.

L'expression (14) devient sensiblement :

$$
n_{c}+n_{a}=\frac{2 \pi}{\mathrm{KRT}} \int_{0}^{x_{1}} q^{2} d x=-\frac{1}{m v_{c}} \int_{q_{0}}^{0} d q=\frac{q_{0}}{m v_{c}},
$$

et par suite on a, comme si la charge était superficielle,

$$
n_{c}=\frac{q_{0}}{m v_{c}}, \quad n_{a}=0
$$

Ces formules ne sont pas rigoureuses, car en profondeur il y a toujours des couches où $\mathrm{U}_{c}$ et $\mathrm{U}_{a}$ ne sont pas tels que l'approximation soit permise, mais l'erreur relative tend vers zéro,quand la charge croît sans limite.

Ainsi, lorsque la charge devient très grande, les couches les plus voisines de la surface tcindent à devenir prépondérantes. Ces fortes charges n'existent du reste que dans les cas de la polarisation ou des différences de potentiel au contact.

Les formules doivent être interprétées avec précaution lorsqu'on est amené à considérer des intervalles moindres que les distances moléculaires.

11. Corps très pauvre en ions. - 11 faut rechercher vers quelles limites tend la formule quand $\mathrm{C}$ tend vers zéro, la charge $q_{0}$ r estant invariable. Comme dans le cas précédent, un des U (par exemple $\mathrm{U}_{c}$ ), devient très grand. D'après $(13)$, on a sensiblement :

$$
\varepsilon=\frac{1}{q_{0}} \frac{\mathrm{KRT}}{4 \pi m v_{c}}\left(\log q_{0}^{2} \frac{2 \pi v_{c}}{\mathrm{KRT}}+\log \frac{1}{\mathrm{C}}\right) .
$$

Ainsi $\varepsilon$ finit par être proportionnel à $\log \frac{1}{\mathrm{C}}$ et crôit donc sans limite.

La densité $\rho$, là où un des $\mathrm{U}$ est grand, ne dépend plus de $\mathrm{C}\left({ }^{1}\right)$.

(1) Il n'y a pas de contradiction entre ces deux faits. Les couches les plus profondes, bien qu'ayant de faibles charges, sont importantes dans le calcul de $\varepsilon$, à cause du facteur $x$ qui entre dans son expression.

Il faut remarquer aussi que le liquide doit toujours contenir assez d'ions pour subvenir à la charge. 
A la surface même, $\rho_{0}$ vaut $\frac{2 \pi m v_{c}}{\operatorname{KRT}} q_{0}^{2}$, ou $-\frac{2 \pi m v_{a}}{\mathrm{KRT}} q_{0}^{2}$, suivant son signe, ce qui donnerait $9,4 \cdot 10^{3}$ avec une charge d'une unité et les données précédentes.

Le résultat le plus saillant de notre travail est ce point que, dans les liquides peu ionisés, la charge occupe une épaisseur assez sensible (plusieurs microns pour l'eau pure et davantage pour d'autres liquides). Il en résulte que, si une lame isolante de pouvoir inducteur $\mathrm{K}^{\prime}$ sépare deux masses liquides, la capacité du condensateur ainsi formé sera diminuée de ce fait, comme si l'épaisseur du diélectrique était augmentée de $2 \varepsilon \frac{\mathrm{K}^{\prime}}{\mathrm{K}}$. Il est probable que l'expérience serait réalisable avec une lame de mica très mince et un liquide approprié. 